\title{
Evaluation of the Factors Related to the Pain by Fractional Energy Delivery System
}

\author{
Boncheol Leo Goo ${ }^{1,2}$ \\ Kee-Yang Chung ${ }^{2}$ \\ ${ }^{1}$ Clinique L, Goyang, Korea \\ ${ }^{2}$ Department of Dermatology, Yonsei University \\ Graduate School of Medicine, Seoul, Korea
}

\author{
Background and Objectives \\ Fractional energy delivery devices are capable of providing different \\ arrangement of spots or columns that break the skin. Sensory function of \\ human skin can be used to detect the geographic pattern of pain more \\ crudely compared to other tactile senses.
}

\section{Materials and Methods}

Ten subjects were treated with a fractional microneedle radiofrequency device, which was capable of providing varying voltage and pulse duration at different adjustable penetration depth. To assess the characteristics of pain from different arrangements of the energy delivery spots, different conditions were analyzed in terms of column arrangement.

\section{Results}

The degree of pain was proportionally related to the increase in voltage, penetration depth, and total treatment area, and inversely proportional to the increase in pulse duration. No difference was observed when different types of microneedle arrays were used in the same amount of treatment area.

\section{Conclusion}

The finding of this study implies that the arrangement of energy spots can be regulated to decrease the pain in the energy device treatment, which can be useful for the development of the devices.

\section{Key words}

Fractional energy delivery devices; Pain; Gate theory; Microneedle 


\section{INTRODUCTION}

Fractional energy delivery devices have been used for various skin conditions for more than a decade. Practice using these devices made a remarkable progress in scar revision and skin rejuvenation. Nevertheless the pain from deep-penetrating, fractionated pattern of wound by energy- delivery devices often lessen the compliance of the patients, which continues to give bad influence to the success of treatment eventually.

Because the amount of energy itself is to be decided by the surgeons with clinical decision, most of the challenges to decrease the pain and other side effects are focused on the pattern design in the development of the fractionated energy delivery system. Many factors are to be postulated, nevertheless not many of the factors have been analyzed.

Peripheral sensory system of human can detect the sensation site only with limited 'resolution' by the different organs. Even in the same organ, the resolution or discrimination ability of the pain sites can be remarkably different by location. For example, closely located different pinch or sting sites within $1 \mathrm{~mm}$ usually cannot be discriminated in the back, but it can be discriminated in reading braille with fingertips. On much cruder senses like pain, not many of researches have been done for its discrimination limits, comparing to other tactile functions of human. Especially in the medical lasers or energy delivery devices, even though these devices are usually causing significant pain, researches have been focused on the relief of the pain itself with other methods or technology, without evaluation of the characteristic analysis.

Subject of this study was to assess the factors involved in the pain from fractional energy delivery devices.

\section{MATERIALS AND METHODS}

The study design was reviewed and confirmed by ethics board of Clinque L Dermatology (Goyang, Korea) and Lutronic corporation (Goyang, Korea). 10 healthy adults were enrolled voluntarily. Exclusion criteria was as following: unwanted volunteering, any history of dermatologic illnesses or diseases including contact dermatitis, urticaria, and active or chronic infectious status, fear of needles and related psychosomatic symptoms, tendency of bleeding or other hematologic disorders.

On the ventral side of forearms, treatment area was marked as described in Fig. 1. Topical anesthesia was achieved with application for 20 minutes with $2.5 \%$

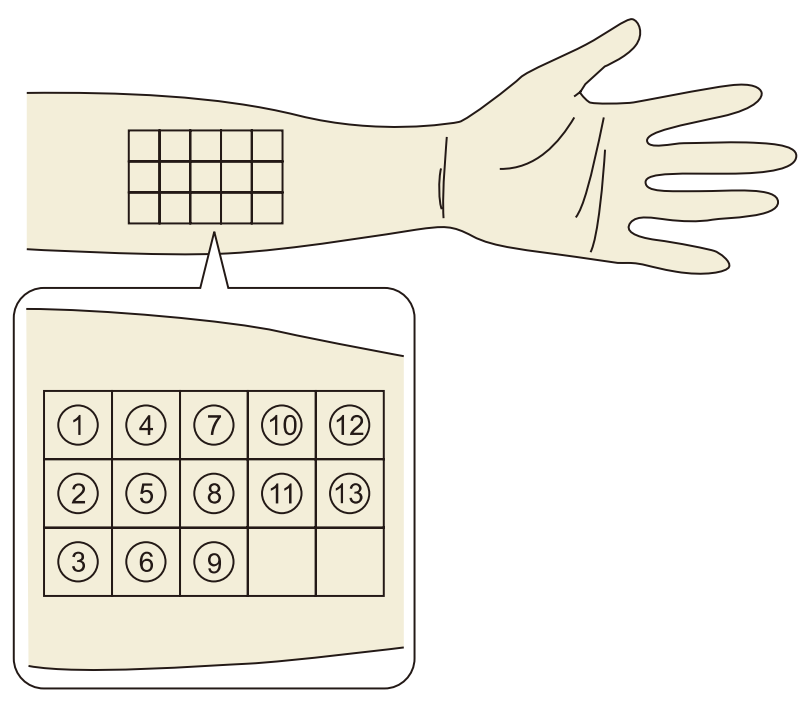

Fig. 1. Design of treatment lesion.

lidocaine and 2.5\% prilocaine cream (EMLA). Each grid was treated with single shot of microneedle fractional radiofrequency device $\left(I N F I N I^{T M}\right.$, Lutronic Corporation, Goyang, Koreal. The parameter was changed at each grid to assess different factors. The study design was reviewed and confirmed by ethics boards of committee of Clinque $L$ Dermatology and Lutronic corporation.

All parameters were selected within the safety range of the treatment parameter for skin rejuvenation from the manufacturer. To assess each factor, other parameters were fixed at the same values (Table 1).

Pain at different energy levels (Voltage) was measured at 4,6 and 8 (fixed pulse duration $(60 \mathrm{~ms}$ ) and penetration depth $(1.5 \mathrm{~mm})$. Each difference between the level was 2.5 $V$ in voltage. Pulse duration (millisecond) 40, 80, 120 (fixed energy level at 5 , penetration depth at $1.5 \mathrm{~mm}$ ). Needle penetration depths $(\mathrm{mm})$ were compared in 0.5, 1.5 and $2.5 \mathrm{~mm}$ (fixed energy level at 5 , pulse duration at $60 \mathrm{~ms}$ ). All conditions were treated with same type of tip with same arrangement of needles, 49 needles at $1.21 \mathrm{~cm}^{2}$ treatment area.

Pain at different arrangement of needles was measured. Pain with different numbers of needles per area (16/49 per $\left.1.21 \mathrm{~cm}^{2}\right)$ was assessed, and the difference in the pain due to different sizes of total treatment area $10.2704 \mathrm{~cm}^{2}, 1.21 \mathrm{~cm}^{2}$ with 16 needles, different gap distances) was measured (Fig. 2). All other parameters were fixed as same lenergy level at 5 , pulse duration at 60 $\mathrm{ms}$, penetration depth at $1.5 \mathrm{~mm}$ ).

In the treatment, each subjects was blinded with eye covers, not to detect the difference in parameters and 
Table 1. Treatment condition

\begin{tabular}{cccccc}
\hline Grid no. & Voltage $(\mathrm{V})$ & Pulse duration $(\mathrm{ms})$ & Needle depth $(\mathrm{mm})$ & Number of needles (n) & $\begin{array}{c}\text { Size of Tx. area } \\
\text { (square centimeter) }\end{array}$ \\
\hline 1 & 10 & 60 & 1.5 & 49 & 1.21 \\
2 & 15 & 60 & 1.5 & 49 & 1.21 \\
3 & 20 & 60 & 1.5 & 49 & 1.21 \\
4 & 12.5 & 40 & 1.5 & 49 & 1.21 \\
5 & 12.5 & 80 & 1.5 & 49 & 1.21 \\
6 & 12.5 & 120 & 1.5 & 49 & 1.21 \\
7 & 12.5 & 60 & 0.5 & 49 & 1.21 \\
8 & 12.5 & 60 & 1.5 & 49 & 1.21 \\
9 & 12.5 & 60 & 1.5 & 49 & 1.21 \\
10 & 12.5 & 60 & 1.5 & 49 & 1.21 \\
12 & 12.5 & 60 & 1.5 & 1.5 & 1.21 \\
13 & 12.5 & 60 & 1.5 & 16 & 0.2704 \\
14 & 12.5 & 60 & & 1.21
\end{tabular}

*Characteristics to be compared were marked in red.
$\begin{array}{llllllllllll}0 & 0 & 0 & 0 & 0 & 0 & 0\end{array}$
$\begin{array}{lllllllllllllll}0 & 0 & 0 & 0 & 0 & 0 & 0\end{array}$
0000000
$\begin{array}{lllllllllllllllll}0 & 0 & 0 & 0 & 0 & 0 & 0\end{array}$
$0 \quad 0 \quad 0 \quad 0 \quad 0 \quad 0 \quad 0$
$0 \quad 0 \quad 0 \quad 0 \quad 0 \quad 0 \quad 0$
0000000

49 needles, $1.21 \mathrm{~cm}^{2}$

16 needles, $0.2704 \mathrm{~cm}^{2}$

assessment was done only verbally. Parameters were spoken numerically symbolized (e.g. "Condition $1^{\text {st }}$ is to be shot"). For each condition, numeric (1 to 10) visual analogue scale (VAS) was recorded for the intensity of pain. Modified McGill's questionnaire ${ }^{1}$ (exclusively selected questionnaires in the sensory charateristics "what does your pain feel like?" translated into PRI (Pain Rating Index 0 to 78). All volunteers were informed of possible side effects and complications and monitored with planned visit for follow-up.

Statistical analysis were measured by student's t-test using SPSS14 (IBM SPSS statistics, IBM corporation, Armonk, NY, USA) and the values were regarded as significant as $p$-value $<0.05$.

\section{RESULTS}

Total 10 volunteer healthy subjects ( 3 males, 7 females) were screened and enrolled and finished the study. Side

$\begin{array}{llll}0 & 0 & 0 & 0 \\ 0 & 0 & 0 & 0 \\ 0 & 0 & 0 & 0 \\ 0 & 0 & 0 & 0\end{array}$

16 needles, $1.21 \mathrm{~cm}^{2}$
Fig. 2. Arrangement of microneedles (diameter: 200 micrometers) in the tips used for the study: 49 tip (1.21 $\left.\mathrm{cm}^{2}\right) / 16$ tip $\left(0.2704 \mathrm{~cm}^{2}\right) / 16$ tip $\left(1.21 \mathrm{~cm}^{2}\right)$.

effect was monitored as minimal as hours of partiall erythema on the lesion. No complication was observed on planned follows-up.

In the assessment of pain due to different energy levels (voltage), gradual increase was statistically significant by increasing energy levels (Fig. 3).

In the assessment of pain due to different pulse durations, gradual decrease was observed by longer pulse duration, resulted as not significant statistically (Fig. 4).

In the assessment of pain due to the penetration depth, an increase tendency was prominent and statistically significant (Fig. 5).

In the assessment of pain due to the needle numbers, different numbers of needles in certain fixed area didn't show significant difference in pain (Fig. 6).

In the assessment of total treatment area, larger size or larger needle assorted tip showed statistically significant increase of pain (Fig. 7).

In the assessment of detailed pain characteristics in 
A

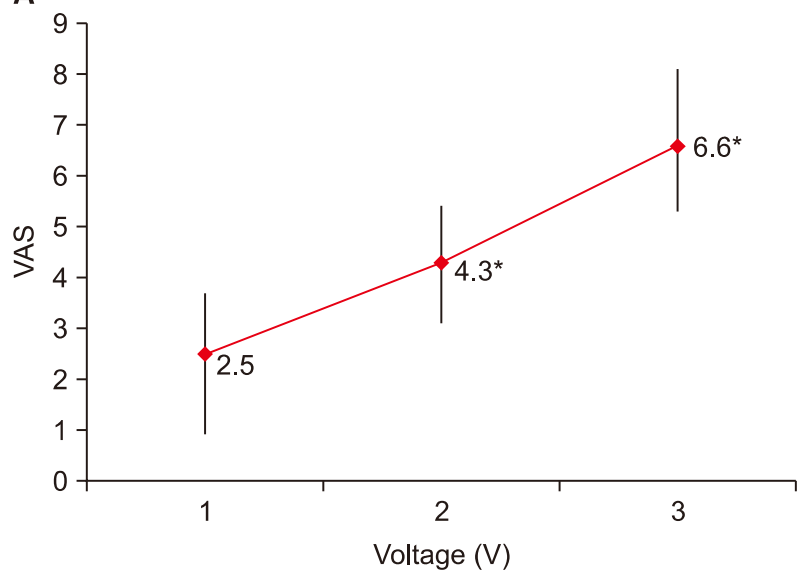

B

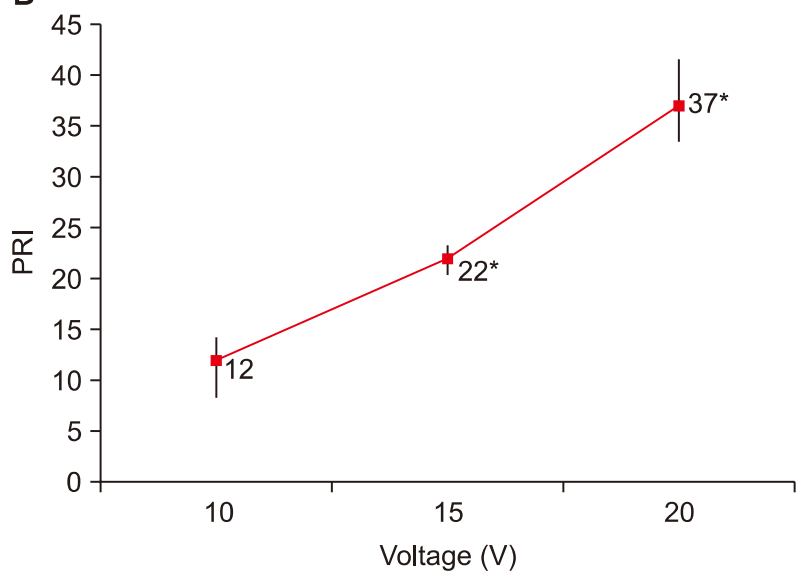

Fig. 3. Change of pain degrees due to the energy level increases. (A) VAS. (B) PRI.

A

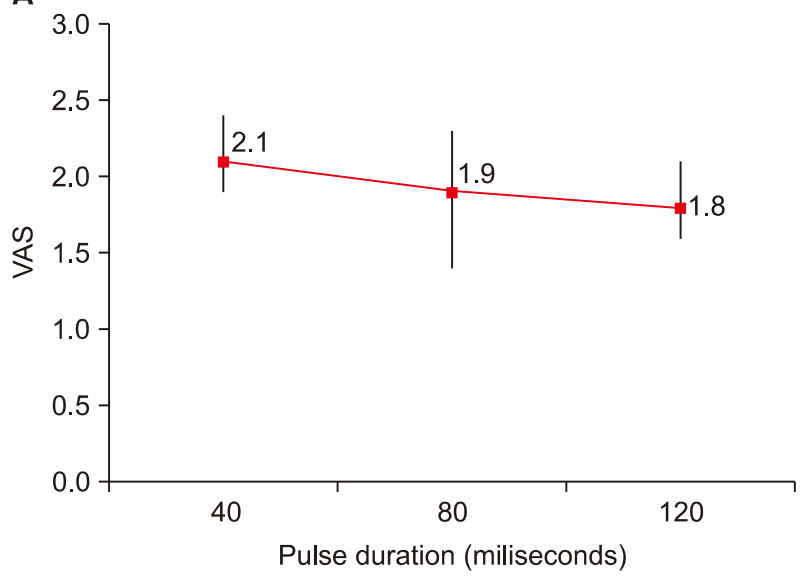

B

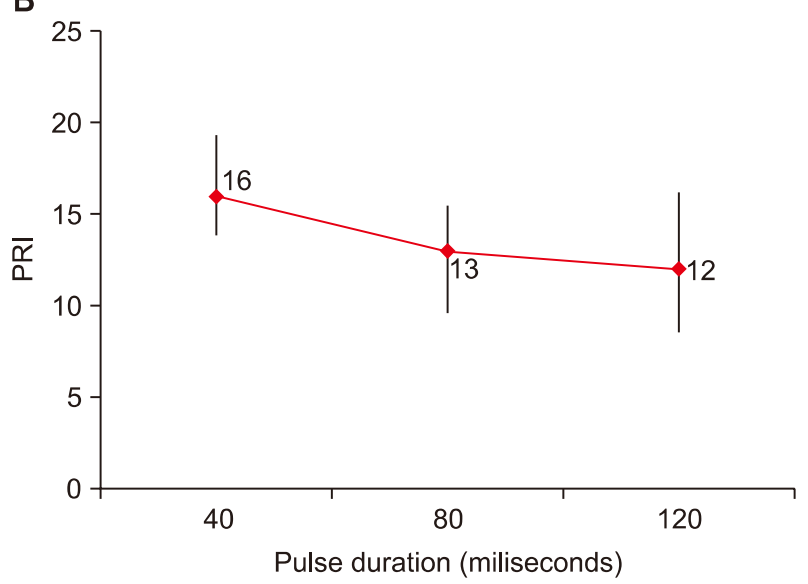

Fig. 4. Change of pain degrees due to the pulse duration increase. (A) VAS. (B) PRI.

A

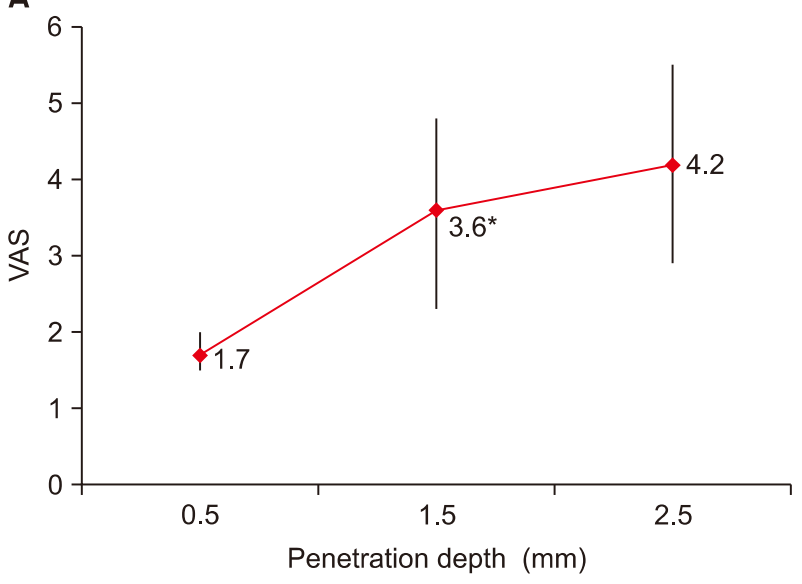

B

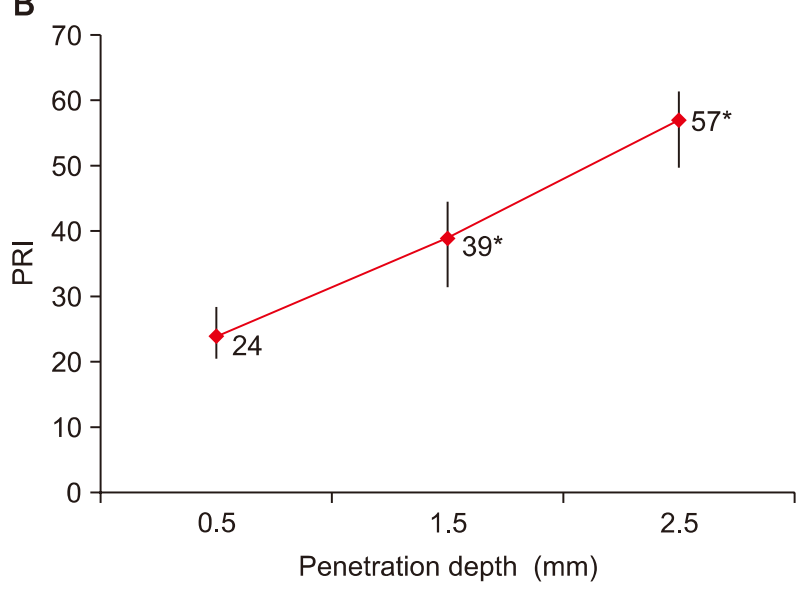

Fig. 5. Change of pain due to the penetration depth increase. (A) VAS. (B) PRI. 


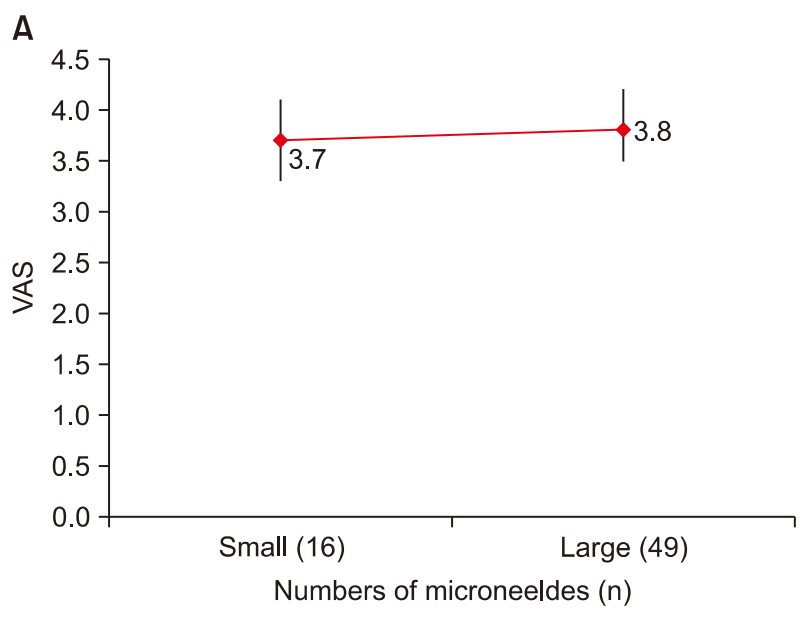

Fig. 6. Change of pain due to the needle numbers. (A) VAS. (B) PRI.

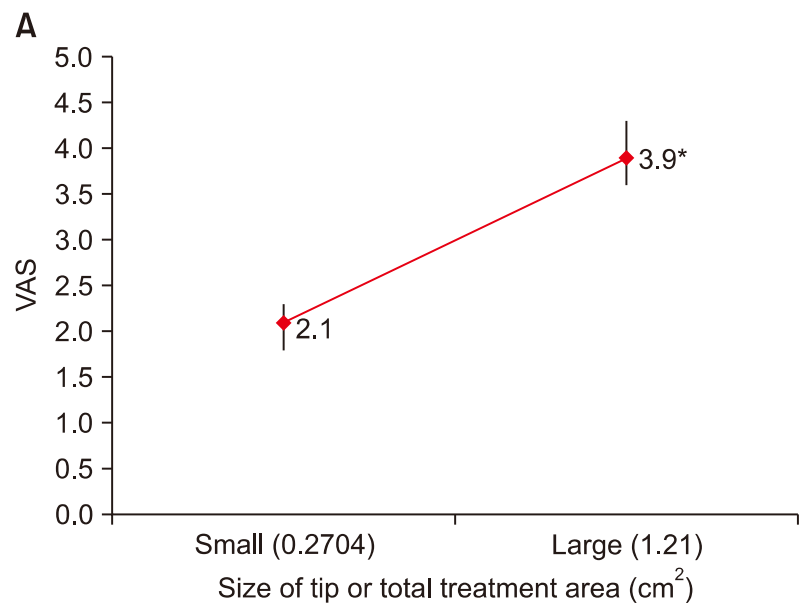

Fig. 7. Change of pain due to the total treatment area. (A) VAS. (B) PRI.
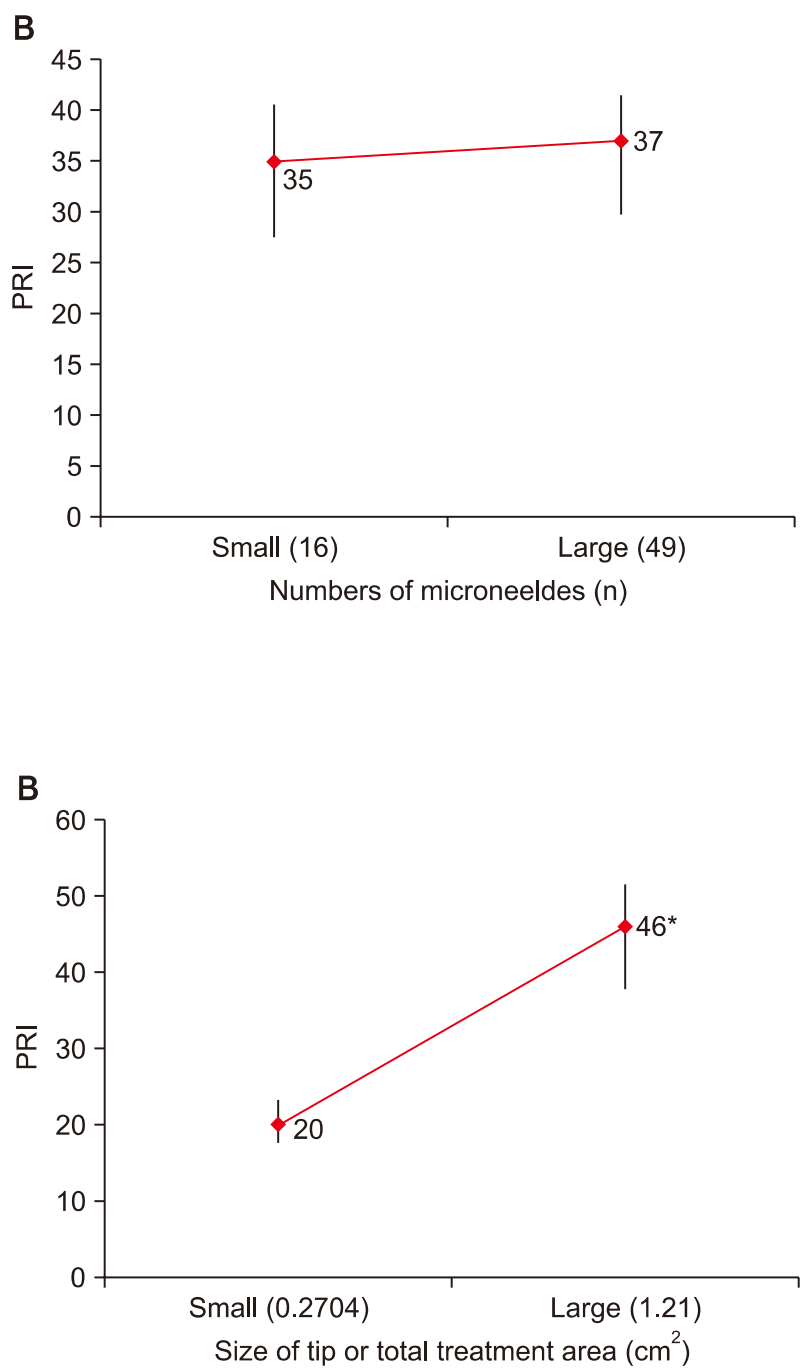

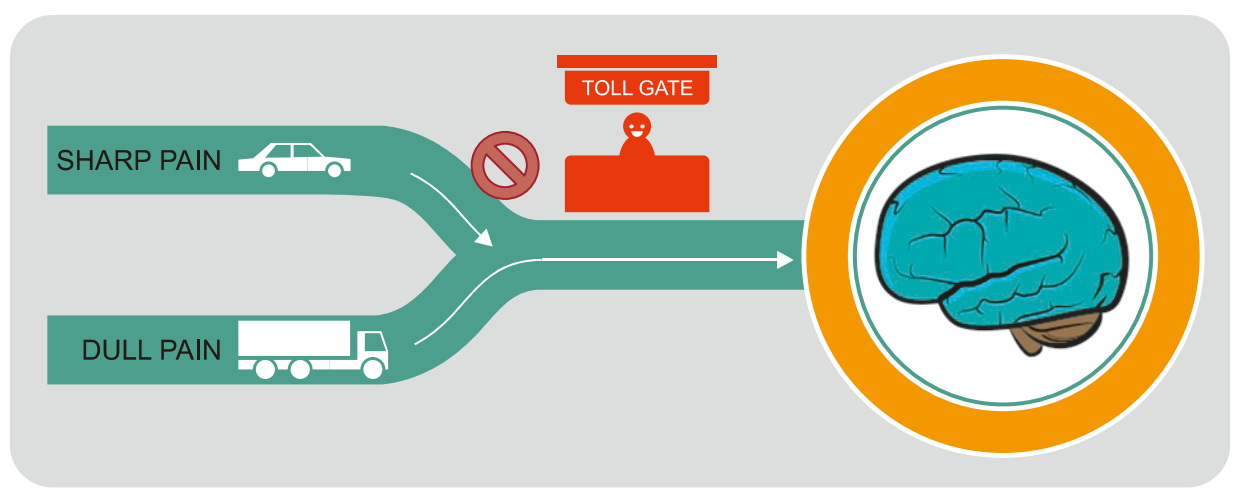

Fig. 8. Schematic explanation of gate control theory in pain signaling. When higher stimulation of other sensation happens, the other sensation or transmission is blocked on periphery, results in delayed or decreased delivery to the central nervous system.
McGill questionnaire, only the tendency toward more 'spreading' type of pain and increase of 'heat sensation' in reverse proportion by longer pulse duration was observed.

\section{DISCUSSION}

Pain by energy delivery device can be an obstacle in the treatment. Despite of significant improvement in 
various anesthesia methods, it is still a challenge for the physicians or surgeons in many in-office setting procedures using energy delivery devices.

Fractionated energy delivery devices are widely used for skin rejuvenation and other treatments in both cosmetic and medical applications. The pattern of fractionation is emphasized when it comes to the complications because of its potential to give excessive energy in to the skin, mostly issued as an overt accumulation of heat. Sequential stimulation of sensory organs can modify the intensity of signal transduction in peripheral nervous system. Cold sensation is widely used stimulation to mask or avoid the following pain sensation. The number, density, depth, and spot arrangements are to be considered with this concept. Usually these factors are considered as simple as contributing in proportionately, otherwise this study showed the arrangement or the location of spots could regulate the actual sensation resulted in a different perception in the central nervous system.

Gate control theory of the pain sensation has been discussed for a long time for explaining the competition or masking of one sense to other. ${ }^{2,3}$ Sequential stimulation of sensory organs can modify the intensity of signal transduction in peripheral nervous system (Fig. 8). Coldness or vibration sensation is widely used stimulation to mask or avoid the following pain sensation. This theory was partially applied to the certain medical devices like transcutaneous electrical nerve stimulation (TENS) system to avoid unwanted pain from medical illnesses or planned treatment like radiofrequency devices for skin rejuvenation. ${ }^{4}$ For instance, 'flow needling' technology using sequential shooting of needles enables smooth insertion into the skin, and relief of pain by 'row-by-row' stimulation of adjacent tissues. ${ }^{5}$

In fractional microneedling energy device, this compensation can be estimated as stronger because the insertion of microneedles is done by mechanical impact, driven by the motorized shooting action of needles. It results in two-step acquisition of sense-dull pain from the mechanical impact, and sharp pain turned from the heat generation in short time. Because the device used in this study applied the needles with insulated body, heat generation was confined at the tip of microneedles, so the sharp pain near the surface through more of unmyelinated small c fibers could be blocked. In slow motion of needle insertion, this can be weakened and to be clarified. For other fractional energy delivery devices like non-ablative or ablative fractional lasers giving the energy constantly from the surface, this finding can appear less.

The limitation of this research is the anlaysis was based on the subjective measurement. Blinding was done to the subjects, while not to the rator. To assess different type of arrangement or sequences, more sophisticated design and larger scale study will be required.

\section{ACKNOWLEDGMENTS}

Jae-Hyun Hwang (Medical and Scientific Affairs, Lutronic corporation) contributed in his illustrations of this paper.

\section{REFERENCES}

1. Melzack R. The McGill Pain Questionnaire: major properties and scoring methods. Pain 1975;1:277-99.

2. Melzack R, Katz J. The gate control theory: reaching for the brain. In: Graig KD, Hadjistavroppulost T, editors. Pain: Psychological Perspectives. 1st ed. Mahwah: Lawrence Erlbaum; 2004. p.36-44.

3. Skevington S. Introduction In: Skevington S, editor. Psychology of Pain. 1st ed. Chichester: Wiley; 1995. p.11-25.

4. Robinson AJ. Clinical Electrophysiology: Electrophysiology and Electrophysiologic Testing. 3rd ed. Philadelphia: Lippincott Williams and Wilkins; 2007. p.123-39.

5. Kim BJ et al. Needle tips for high frequency treatment, high frequency treatment apparatus using same, and high frequency therapy. Patent W02012144713A1 (A61N1/04-06, A6H39/04) 2012 Available from: URL http://www.google.com/ patents/W02012144713A1?cl=en. 